E3S Web of Conferences 4, 03002 (2014)

DOI: $10.1051 / \mathrm{e} 3$ sconf/20140403002

(C) Owned by the authors, published by EDP Sciences, 2014

\title{
The simple dark matter search: Present and future
}

\author{
M. Felizardo ${ }^{1,2}$, T.A. Girard ${ }^{2}$, A.C. Fernandes ${ }^{2,3}$, T. Morlat ${ }^{4}$, J. Puibasset ${ }^{5}$, A.R. Ramos $^{2,3}$, \\ J.G. Marques ${ }^{2,3}$, A. Kling ${ }^{2,3}$, F.P. Carvalho ${ }^{3}$, M.I. Prudencio ${ }^{3}$, A. Gouveia ${ }^{3}$, R. Marques ${ }^{3}$, M. Auguste ${ }^{6}$, \\ D. Boyer ${ }^{6}$, A. Cavaillou ${ }^{6}$, J. Poupeney ${ }^{6}$ and C. Sudre ${ }^{6}$ \\ ${ }^{1}$ Department of Physics, Universidade Nova de Lisboa, 2829-516 Monte da Caparica, Portugal \\ ${ }^{2}$ Centro de Física Nuclear, Universidade de Lisboa, 1649-003 Lisboa, Portugal \\ ${ }^{3}$ Instituto Tecnológico e Nuclear, Instituto Superior Técnico, Universidade Técnica de Lisboa, EN 10, \\ 2686-953 Sacavém, Portugal \\ ${ }^{4}$ École Normale Supérieure de Montrouge, 1 rue Maurice Arnoux, 92120 Montrouge, France \\ ${ }^{5}$ Centre de Recherche sur la Matière Divisée, CNRS et Université d'Orléans, 45071 Orléans, \\ France \\ ${ }^{6}$ Laboratoire Souterrain à Bas Bruit (Université de Nice), 84400 Rustrel-Pays d'Apt, France
}

\begin{abstract}
We review the recently completed Phase II measurements of the SIMPLE project, and briefly describe its in-progress Phase III.
\end{abstract}

\section{Introduction}

The customarily recognized underground physics is in recent times joined by astroparticle physics activities, by virtue of the rock overburden which reduces the cosmogenic radiation backgrounds that would otherwise make its experiments unfeasible. SIMPLE is one such activity, which searches for evidence of the dark matter of the universe in the form of nuclear recoil events generated by the elastic scattering of weakly interacting massive particles (WIMPs) with target nuclei. Its recently completed Phase II measurements, following some 10 years of R\&D activity in the Laboratoire Souterrain à Bas Bruit (LSBB) [1], have yielded exciting new results in this search [2, 3].

\section{Phase II review}

The Phase II [2, 3] measurements were conducted in two Stages, between 27 October 2009 - 5 February 2010 and 12 April-22 July 2010, each comprising 15 high concentration superheated droplet detectors (SDDs), $1-2 \%$ suspensions of micrometric superheated liquid $\mathrm{C}_{2} \mathrm{ClF}_{5}$ droplets in a viscous-elastic $900 \mathrm{ml}$ gel matrix, which undergo transitions to the gas phase upon energy deposition by incident radiation. The physics of the superheated liquid response to incident radiation dictates that [4]: (i) the energy deposited be greater than a thermodynamically defined minimum energy, and (ii) this energy be deposited within a thermodynamically defined minimum distance inside the droplet. Together, energy depositions of order

This is an Open Access article distributed under the terms of the Creative Commons Attribution License 4.0, which permits unrestricted use, distribution, and reproduction in any medium, provided the original work is properly cited. 


\section{E3S Web of Conferences}

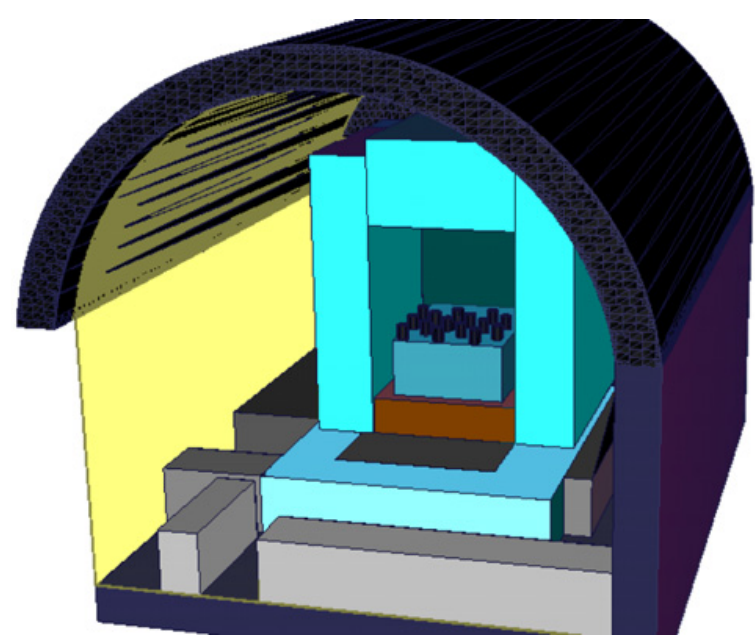

Figure 1. Schematic setup of the Phase II SIMPLE experiments, showing the SDDs (dark blue) in their water pool (medium blue) within GESA, surrounded by water (light blue) and wood + PE shielding (brown).

$\sim 150 \mathrm{keV} / \mu \mathrm{m}$ are required for a bubble nucleation, which renders the technique effectively insensitive to the majority of traditional light particle backgrounds (including electrons, $\gamma$ 's and cosmic muons) which complicate more conventional dark matter search detectors. Both $\alpha$ 's and neutron-generated recoil events fulfill the two criteria, and constitute the principle experimental backgrounds.

The SDDs were operated in the 1500 mwe deep, $60 \mathrm{~m}^{3}$ GESA facility of the LSBB. The cavern is shielded from the neutrons of the rock environment by a $30-100 \mathrm{~cm}$ thickness of concrete, which is internally sheathed by a $1 \mathrm{~cm}$ thickness of iron, as seen in Fig. 1. The SDDs were immersed in a temperature-controlled, 700 liter water pool within the cavern, which rested on a dual vibration absorber placed atop a $30 \mathrm{~cm}$ thick wood platform, resting on a $50 \mathrm{~cm}$ thick concrete floor. The pool was surrounded by layers of sound and thermal insulation. An additional $50-75 \mathrm{~cm}$ thick water shielding surrounded the pool and platform, with a $75 \mathrm{~cm}$ water thickness overhead; $50 \mathrm{~cm}$ of water separated the pool bottom from the detector bases.

The ambient radon level varied seasonally as a result of water circulation in the mountain, and is of concern because of its $\alpha$-generating decay chains. The level was both monitored, and reduced during measurements by replacing the cavern air at $\sim 0.2 \mathrm{~m}^{2} \mathrm{~s}^{-1}$ and by circulation of the water-pool at $25 \mathrm{liter} / \mathrm{min}$ (equivalent to replacing the top $1 \mathrm{~cm}$ water layer each minute) through the temperaturecontrolled external cryo-thermostat; the $(\alpha, n)$ contribution was further reduced by the short radon diffusion lengths of the SDD construction materials (glass, plastic, metal), the SDD $\mathrm{N}_{2}$ over-pressuring, and the glycerin layer covering the SDD gel matrix.

Monte-Carlo simulations of the on-detector neutron field [5], based on radio-assays of the shielding materials and accounting for spontaneous fission, decay- induced $(\alpha, \mathrm{n})$ reactions and $(\mu, \mathrm{n})$ reactions in the rock, showed negligible variations for concrete thicknesses $\geq 20 \mathrm{~cm}$. The Stage 1 shielding was augmented in Stage 2 by an additional $20 \mathrm{~cm}$ thickness of poly ethylene directly below the water pool, reducing the expected neutron background by a factor 3 to $0.33 \pm 0.001$ (stat) \pm 0.038 (syst) evt $/ \mathrm{kgd}$.

Each SDD was fabricated in the 210 mwe underground clean room, following previously described, standard procedures [6], then transported to GESA where it was capped with an electret microphone [7], pressurized to $2.00 \pm 0.05 \mathrm{bar}$, and immersed in the water pool held at $9.0 \pm 0.1^{\circ} \mathrm{C}$.

Stage 2 obtained a total exposure of $13.67 \mathrm{kgd}$, as a result of the detector installation protocol and mechanical failure of 4 SDDs during the run because of over-pressuring; no weather-induced data losses 


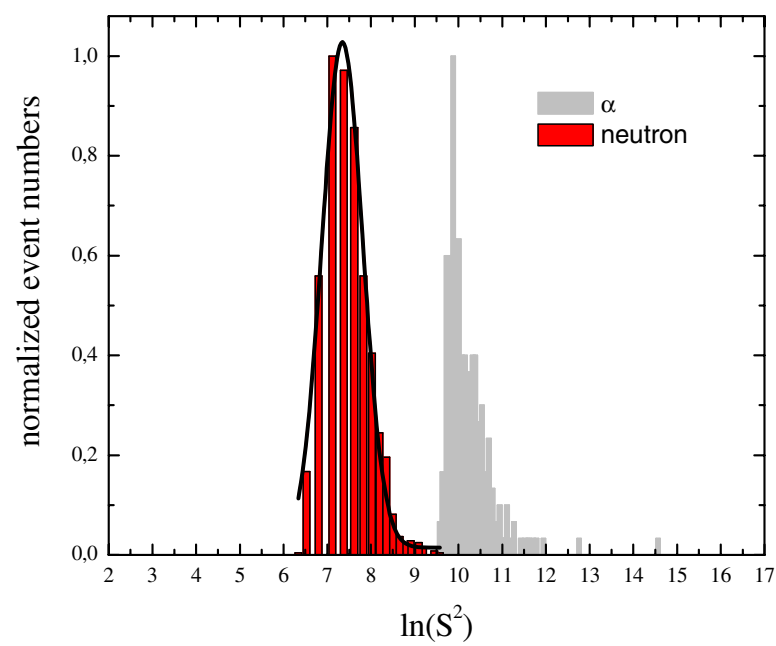

Figure 2. Histogram of neutron-induced nuclear recoil and $\alpha$-induced events as a function of the event amplitude.

occurred, in contrast to Stage 1 which obtained a total of $13.47 \mathrm{kgd}$ of data as a result of frequent, short term power failures during December and January. The SDD pressures in Stage 2 were however allowed to increase in order to verify the SDD recoil energy threshold $(8.0 \pm 0.1 \mathrm{keV})$, for pressures $\leq 2.2 \pm$ 0.1 bar: together with the loss of 4 devices to over-pressuring induced mechanical failure, the resulting data cut reduced the exposure to $6.71 \mathrm{kgd}$.

The recorded signals were analyzed for acoustic backgrounds, and a particle-induced set extracted in each stage [2, 3], which comprised $\sim 2 \%$ of the respective event records. Neutron- and $\alpha$-calibrations of the SDDs defined a nuclear recoil acceptance window for signal amplitudes (S) with an acceptance of $>99 \%$, in which 10 and 1 recoil events were identified for Stage 1 and 2, respectively.

Since both rates were non-zero but consistent with the neutron background estimates, upper limits on the possible number of WIMP events in the data set was calculated for a $90 \%$ C.L. for each measurement Stage by applying the Feldman-Cousins method [8], based on the observation of $\mathrm{j}$ recoil events against an estimated neutron background of $\mathrm{k}$ events. The results were then merged to obtain an overall rate, which were analyzed via Lewin \& Smith [9] to obtain the impact on the WIMP phase space.

The results are presented in Figs. 3 and 4, together with those from other leading search together with leading searches, in the form of standard exclusion contours at a 90\% C.L. where $\sigma$ is the relevant WIMP interaction cross section and $\mathrm{M}_{\mathrm{W}}$ is the WIMP mass; in general, the area above a contour is excluded by the search experiment, with the area below unexplored. Aside from providing the most restrictive limits below $60 \mathrm{GeV} / \mathrm{c}^{2}$ on a possible spin-dependent WIMP-proton coupling to date (Fig. 3), they intersect for the first time with results from IceCube and SuperKamiokande (shown as broken contours). Also note that of the three direct search contours shown, two are from the competitive North American PICASSO and COUPP searches which also use superheated liquids (with substantially larger exposures).

In contrast, the spin-independent results (Fig. 4), while lacking the exposures and coherent $\sigma$ enhancement of their heavier, more sensitive counterparts, join XENON and CDMS in discounting a significant part of the recent low WIMP mass region of current interest identified by the closed contours of CoGeNT, CRESST and DAMA/LIBRA. This is because of the relatively low recoil energy threshold of SIMPLE, which flattens the contour at low $\mathrm{M}_{\mathrm{W}}$ to provide an increased sensitivity. 


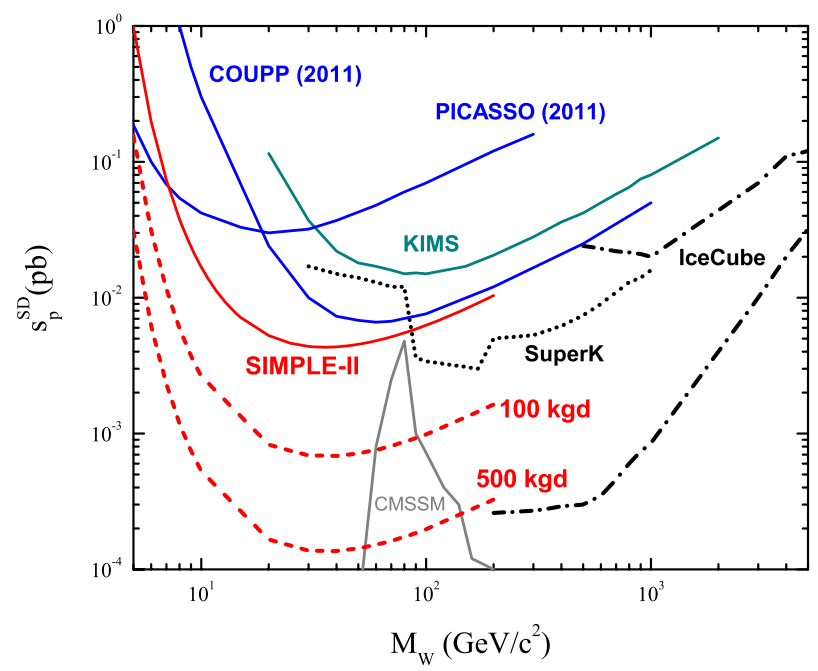

Figure 3. Current results and Phase III sensitivity levels in the spin-dependent sector, with the areas above the contours being excluded. The contours labelled 100 and $500 \mathrm{kgd}$ represent the sensitivities expected in Phase III from the corresponding exposures. The area labelled "CMSSM" represents the theoretically-suggested region allowed by the constrained minimal supersymmetric model.

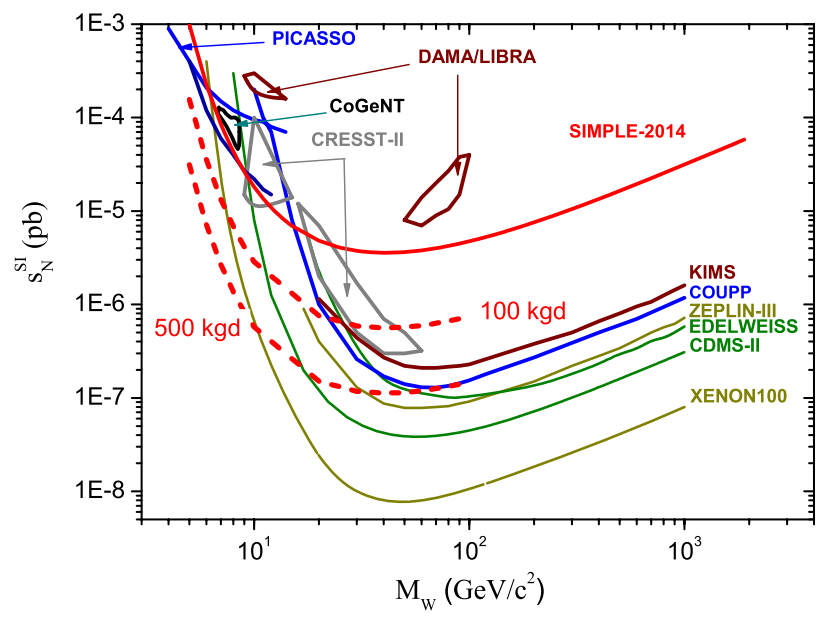

Figure 4. Impact of current results in the spin-independent sector, the areas above the contours being excluded. The enclosed areas indicate the regions currently identified by CoGeNT, CRESST and DAMA/LIBRA within which possible light mass WIMPs are not excluded. The contours labelled 100 and $500 \mathrm{kgd}$ represent the sensitivities expected in Phase III with the corresponding exposures.

\section{Phase III}

The active target mass of SDDs comprises only 1-2\% of the detector volume, which becomes untenable when envisioning exposures of 100-1000 kgd. Phase III replaces the SDDs with a prototype BC (Bubble Chamber variant): unlike a SDD, a BC involves no distribution of superheated liquid in a gel matrix, so that the device volume $\sim$ active volume. A single $0.5 \mathrm{~kg} \mathrm{BC}$, prototyped by SIMPLE in 2010, differs from more conventional devices in the use of the SIMPLE purified glycerine/food-gel as an internal sheathing of the containment, providing the same smooth freon interface as with the SDDs (to reduce 
the probability of spontaneous nucleation: recompression intervals of up to $\sim 12 \mathrm{~h}$ have been observed), while maintaining the containment radio-purity ( $\alpha$-induced events $<0.5 \mathrm{evt} / \mathrm{kgd}$ ), and preserving the reduced cost of detector fabrication.

Phase III, with a $5 \mathrm{~kg} \mathrm{BC}$, provides $\sim 25 \times$ the active Phase II mass in $\sim$ a quarter of the volume, combined with additional, purified, water neutron shielding. The prototype scale-up to a $5 \mathrm{~kg}$ liquid containment is constrained by commercial plastics and their ability to sustain a 10 bar recompression: at present, a "home water filter" prototype is recompressed using a pressure-activated system, with a new piston-based recompression prototype to separate the recompression gas $\left(\mathrm{N}_{2}\right)$ from the gel and superheated liquid completing development.

The loss of the viscous-elastic gel matrix as the response medium should preclude the use of the previous low frequency instrumentation (since the signal derived from the gel response to a bubble nucleation event): the primary particle-induced event signal obtains [10] from the differing number of proto-bubbles formed in the superheated liquid by recoil ions and $\alpha$ 's, which lie in the high frequency regime of $\sim 130 \mathrm{kHz}$. Recent prototype tests using an externally-polarized condenser microphone with a flat response over $10-150 \mathrm{kHz}$ however surprisingly yielded signals at only $\sim 15 \mathrm{kHz}$, suggesting the continuing effect of the gel/glycerine matrix and enabling the continued use of the previous instrumentation; further study on this issue is in progress.

Phase III is to be executed in GESA, requiring a further decrease in the on-detector neutron flux: we envision an increase of the current water shield thickness to $1.3 \mathrm{~m}$, as constrained by the GESA geometry, with the water replaced by purified reactor containment water which involves a resin-based treatment process which reduces the heavy metals content by $10^{2}-10^{3}$ : a neutron background rate well below $10^{-5} \mathrm{kgd}$ is estimated, with zero neutron-induced background for exposures of $\sim 10^{3} \mathrm{kgd}$.

Estimated Phase III sensitivity levels in the two search sectors with exposures of 100 and $500 \mathrm{kgd}$, using two $5 \mathrm{~kg}$ BC prototypes and assuming the same particle-induced event discrimination as in Phase II (with zero candidate events), are also shown in Figs. 2 and 3. One two-week exposure, assuming a $20 \%$ loss of measurement time due to recompressions, is expected to provide more than a factor 10 improvment in current results.

Assuming the success of Phase III, the project measurements are to be transferred to the Capsule of the LSBB [1] for a Phase IV using twenty 50 liter BCs with a $2 \mathrm{~m}$ all-around purified water shield, with the intent to conclusively observe the elusive WIMP ... or deny its existence.

This work is funded, in part, by the Nuclear Physics Center of the University of Lisbon, and by grants PTDC/FIS/115733/2009 and PDTC/FIS/121130/2010 of the Portuguese Foundation for Science and Technology (FCT). The activity of M. Felizardo is supported by grant SFRH/BD/46545/2008 of FCT.

\section{References}

[1] LSBB http://1sbb.oca.eu

[2] M. Felizardo, et al.: Phys. Rev. Lett. 105 (2010) 211301.

[3] M. Felizardo, et al.: Phys. Rev. Lett. 108 (2012) 201302.

[4] F. Seitz: Phys Fluids 1 (1958) 1.

[5] A.C. Fernandes, et al.: Nucl. Instr. Meth. A 623 (2010) 960.

[6] T. Morlat, et al.: Astrop. Phys. 30 (2008) 159.

[7] M. Felizardo, et al.: Nucl. Instr. Meth. A589 (2008) 72.

[8] G.J. Feldman and R.D. Cousins: Phys. Rev. D57 (1998) 3873.

[9] J.D. Lewin and P.F. Smith, Astrop. Phys. 6 (1996) 87.

[10] F. Aubin, et al.: New Journ. Phys. 10 (2008) 103017. 